East African Medical Journal Vol. 86 May 2009

SUBCLINICAL NEPHROTOXICITY ASSOCIATED WITH OCCUPATIONAL SILICA EXPOSURE AMONG MALE KENYAN INDUSTRIAL WORKERS

D. M. Mwangi, MSc, Assistant Chief Med Lab Technologist, L. J. Njagi, HDMLS, Assistant Chief Med Lab Technologist, Department of Laboratory Medicine, Kenyatta National Hospital, P.O. Box 20723-00202, Nairobi, Kenya, S. O. McLigeyo, MBChB, MMed, Associate Professor, Department of Clinical Medicine and Therapeutics, University of Nairobi, P.O.Box 19676-00202, Nairobi, Kenya, J. M. Kihoro, PhD, Lecturer, Department of Statistics and Actuarial Sciences, Jomo Kenyatta University of Agriculture and Technology, P.O. Box 62000-00200, Nairobi, Kenya, J. J. N. Ngeranwa PhD, Senior Lecturer, G. O. Orinda, PhD, Lecturer, E. N. M. Njagi, PhD, Department of Biochemistry and Biotechnology, Kenyatta University, P.O.Box 43844-00100, Nairobi, Kenya

Request for reprints to: Mr. D. M. Mwangi, Department of Laboratory Medicine, Kenyatta National Hospital, P.O. Box 20723-00202, Nairobi, Kenya

\title{
SUBCLINICAL NEPHROTOXICITY ASSOCIATED WITH OCCUPATIONAL SILICA EXPOSURE AMONG MALE KENYAN INDUSTRIAL WORKERS
}

\author{
D. M. MWANGI, L. J. NJAGI, S. O. MCLIGEYO, J. M. KIHORO, J. J. N. NGERANWA, \\ G. O. ORINDA and E. N. M. NJAGI
}

\begin{abstract}
Objective: To determine early signs of renal injury due to occupational silica exposure. Design: Cross-sectional analytical research.

Settings: Kenyatta National Hospital for the referent population and Clayworks ceramics, bricks and tiles factory for the assessment of occupational silica exposure. Subjects: Thirty three non-smoking silica-exposed male industrial workers and 38 non-smoking male referents participated in this study.

Results: Silica-exposed males excreted significantly increased levels of U.TP, U.Malb, U.ALP, U.y-GT and U.LDH compared to referent males. Among the silica-exposed males, U.Si negatively correlated significantly with age, U.TP correlated significantly to each of U.ALP and U.LDH. However, no correlation was observed between work duration and U.Si.

Conclusion: The present study shows that there is associated glomerular and proximal tubular damage among silica exposed workers which is not duration related and is seemingly subclinical and nonprogressive and urinary silica levels appears to be similar in all groups and are not affected by exposure and work duration: the reason for which is unclear.
\end{abstract}

\section{INTRODUCTION}

Exposure to crystalline silica is classically associated with an inhalation occupational disease called silicosis (1). Industries and occupations having the potential for silica exposure include mining, quarrying, tunneling, foundry work, glass manufacture, abrasive blasting, ceramic, pottery and cement production. The suspicion that silica dust affects the kidney in man is over 80 years old (2). Previous animal studies have demonstrated that silica can produce doserelated nephropathy, causing degenerative changes in tubular epithelium and interstitial inflammation, fibrous nephrosis, glomerulonephritis and systemic vasculitis. For many years, case reports also drew attention to possible nephrotoxic effects of silica and distinct renal histologic alterations in glomerular and proximal tubules in workers exposed to silica (3).

Present clinical and diagnostic screening tests for assessing the functional integrity of kidney include serum creatinine, urea and urinalysis with dipstix. However, these tests are less sensitive for detecting early renal changes by nephrotoxins (4). Various tubular renal urinary biomarkers are also used to study human nephrotoxicity at an early stage (5). Renal urinary biomarkers that are most useful to define defects on various parts of the nephron include the: high molecular-weight protein, albumin for evaluating glomerular integrity; low molecular weight protein; retinol binding protein (RBP) for assessing tubular protein reabsorption, and cytoplasmic enzyme glutathione-S-transferase(GST) in addition to the lysosomal enzyme N-acetyl- $\beta$-Dglucosaminidase (NAG) to indicate tubular injury (3). Damage to tubular epithelial cells causes not only functional problems butalso structural changes, with increased cellular turnover and cell lyses resulting in release of both border cytosolic or lysosomal enzymes. The evaluation of the concentrations of these enzymes in urine is known to be a good and sensitive noninvasive method to measure the tubular cells integrity $(6,7)$. Other urinary renal 
enzymes including gamma glutamyltransferase $(\gamma-$ GT), alkaline phosphatase (ALP) which are found on the epithelial cells of the proximal tubule and the lactate dehydrogenase (LDH) located at distal tubule cells respectively have been used to detect early kidney damage in rats (8). In-spite of their potential applicability little is known about their clinical application in human. The aim of this study was to investigate the effects of occupational silica exposure on sub-clinicalnephrotoxicity by measuring urinary excretion levels of $\gamma \mathrm{GT}$, ALP, LDH and other proteins among Kenyan industrial workers.

\section{MATERIALS AND METHODS}

Study area/study population: The occupational silica exposure subjects were recruited from non-smoking male workers of Clayworks ceramic, brick and tiles factory while the control non-smoking male population were recruited from Kenyatta National Hospital workers who had usual silica exposure sources encountered in daily life, withoutoccupational or other unusual exposures. A questionnaire was used in order to identify the study subject's lifestyle characteristics such as smoking (smoker or nonsmoker), occupational history (silica-exposed or silica non-exposed) and medical history with the medication intake aimed to derive a reconstruction of the subjects' entire occupational career. It was also used to determine the prevalence of indicators of nephrotoxicity in the population during the time of urine collection; the study fitted within the crosssectional subtype of the analytical research study design as described by Mann (9).

Workers were excluded from the study if any was a smoker, had experienced kidney disease or a disease likely to impair renal functions and/or parameters that were to be tested (creatinine, alkaline phosphatase, alanine aminotransferase, aspartate aminotransferase, gamma glutamyltransferase and lactate dehydrogenase). Other exclusion criteria included study subjects who had previous or potential exposure to agents capable of damaging the kidney such as lead, cadmium and mercury or other nephrotoxicants such as organic solvents. The workers could also be excluded if they revealed a history of having consumed drugs with potential nephrotoxicity such as analgesics and anti-inflammatory agents and abuse of aminoglycoside antibiotic therapy, which is associated with renal disorders (10). Those who portrayed results of creatinine of less than $0.3 \mathrm{~g} / 1$ or greater than $3.0 \mathrm{~g} / 1$ were also excluded.

The study subjects were grouped into: silica exposed male workers from Clayworks bricks, ceramic and tiles factory consisting 33 non-smokers; and referent male workers from Kenyatta National Hospital consisting 38 non-smokers.
Ethical consideration: Ethical approval was sought and granted by Kenyatta National Hospital Ethical and Research Committee (KNH-ERC). The participants were reassured of confidentiality in the handling of information and procedures involved in this study. Informed consent was sought from the participants and they were also requested to append their signature on the consent form in order to acknowledge their voluntary approval of participation. Individuals found with evidence of nephrotoxicity at the end of the study were referred for follow up or for further nephrologicevaluation. Theindustry was also advised to institute rotational schedules for staff working in areas with potential occupational silica dustexposure to minimise the risk of kidney damage.

Sample collection: A random single voided, second morning urine sample in a closed container was collected from each participant. The container was labeled with the study number of the participant and the date of birth was also marked to tally into all the required demographic information. This was clearly matched with the demographic information on the questionnaire form to avoid any risk of mix-ups or incorrect identification of samples. The study number was also marked correctly on the bottle and the questionnaire. Spot urine was used because urinary protein/creatinine ratio (11), as well as dividing urinary enzyme activity by the urine creatinine concentration (12) in random sample, correlates with 24 hour urinary excretion and eliminates variations due to changing rates of urine output and provides a measurement of concentration. The collected urine sample was transported in a closed box at room temperature within two hours after the collection.

Sample analysis: Each urine sample was analysed for the assessment of glomerular integrity by measuring urinary levels of total protein (U.TP) using the semiautomated Technicon Bayer RA $1000^{\circledR}$ analyzer (Ireland Technicon Limited) and microalbumin (U.Malb) using Hemocue ${ }^{\circledast}$ urine albumin system (Hemocue AB, Angelhom, Sweden); proximal tubular structural integrity by determining urinary activities of gamma glutamyltransferase(U.y-GT) and alkaline phosphatase (U. $\gamma$ ALP) which are proximal tubule enzymes and lactate dehydrogenase (LDH) located at distal tubule cells (8) using automated Olympus AU640® analyser (Japan Mishima Olympus Optical Company Limited); and urinary silicon levels using the Buck Model 210 VGP Atomic Absorption Spectrophotometer ${ }^{\circledR}$ (Bulk Scientific, Inc).

Internal quality control: The internal quality control sample materials were run together with the urine specimen from the study subjects. Values obtained for the internal quality control materials for each of the 
measured parameters was accepted if it was within plus or minus two standard deviations $( \pm 2 S D)$ from the target value.

Data management and analysis: Results for all the parameters measured were each divided by the levels of the urinary concentration of creatinine in grams per litre (11) in order to correct for variations in urinary concentration due to hydration (13). Data generated from the study was entered into Excel software and then exported to SPSS program version 11.0 for statistical analysis. Data were tabulated as median (range) because most of the measured parameters were not normally distributed. Differences between investigated groups (male referents, male silica exposed subjects) were assessed by Mann-Whitney test. Spearman correlation coefficient was used to examine relationships between studied urinary parameters. Upper limits of normal which were used to calculate the prevalence of abnormally elevated values were defined as 95th percentile of values observed for the referents. These were: U.AST 19.95 U/U.cr; U.ALT,

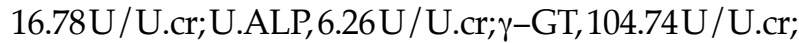

U.LDH, 61.07U/U.cr; U.TP, 150.23 mg/U.cr; U.Malb, $87.24 \mathrm{mg} / \mathrm{U} . \mathrm{cr}$; and U.Si, $13.59 \mu \mathrm{g} / \mathrm{U} . \mathrm{cr}$. Prevalence of abnormal values among silica-exposed groups and referents was compared with Chi-square or Fisher exact tests as appropriate. Results were considered statistically significant at $\mathrm{p}<0.05$.

\section{RESULTS}

Effects of silica exposure on proteinuria and enzymuria of male industrial workers: Table 1 and 2 shows the effect of silica exposure on proteinuria and enzymuria of male industrial workers and the prevalence of abnormally elevated values, respectively. Results show that: silica exposed male industrial workers had a significantly increased U.TP $(p=0.017)$, U.Malb ( $p$ $<0.001)$, U.ALP ( $p<0.001)$, U. $\gamma$-GT $(p<0.001)$ and U.LDH $(\mathrm{p}<0.001)$, relative to that of referent male workers; the prevalence of abnormally elevated values of U.TP $(p<0.001), U . A L P(p=0.003), U . \gamma-G T(p=0.035)$ and U.LDH $(p<0.001)$, is significantly greater in silica exposed male industrial workers relative to that of referent male workers.

Table 1

Proteinuria and enzymuria among silica exposed male workers

\begin{tabular}{lcc}
\hline Parameter & $\begin{array}{c}\text { Referent (n=38) } \\
\text { Median (Range) }\end{array}$ & $\begin{array}{c}\text { Silica exposed (n=33) } \\
\text { Median (Range) }\end{array}$ \\
\hline Age (years) & $37.5(27-53)$ & $43.5(21-56)$ \\
Work duration (years) & $15.0(3-30)$ & $23.3(1-31)$ \\
U.AST/U.cr (U/gU.cr) & $8.16(2.48-30.72)$ & $9.05(1.26-88.04)$ \\
U.ALT/U.cr (U/gU.cr) & $6.89(0.00-18.29)$ & $6.90(0.00-53.76)$ \\
U.ALP/U.cr (U/gU.cr) & $1.78(0.00-11.43)$ & $4.13(0.65-32.01)^{\mathrm{a}}$ \\
U.y-GT/U.cr (U/gU.cr) & $36.10(13.72-122.14)$ & $66.03(29.42-490.90)^{\mathrm{a}}$ \\
U.LDH/U.cr (U/gU.cr) & $24.23(16.89-54.03)$ & $54.91(27.64-410.86)^{\mathrm{a}}$ \\
U.TP/U.cr (mg/gU.cr) & $22.62(4.84-186.80)$ & $51.07(15.08-2156.00)^{\mathrm{a}}$ \\
U.Malb/U.cr (mg/gU.cr) & $0.97(0.40-111.24)$ & $14.05(0.62-140.02)^{\mathrm{a}}$ \\
U.Si/U.cr (mg/gU.cr) & $5.92(0.00-17.43)$ & $5.38(1.15-36.80)$ \\
\hline
\end{tabular}

Results are expressed as median (Range) ${ }^{a} \mathrm{p}<0.05$ for referent male workers compared to silica exposed male workers.

Table 2

Prevalence of abnormally elevated values of measured urinary parameters in referents and silica exposed male workers

\begin{tabular}{lccccc} 
Parameter & Referents $(\mathrm{n}=38)$ & Silica exposed & workers $(\mathrm{n}=33)$ & P-value \\
& No. & $(\%)$ & No. & $(\%)$ & \\
\hline U.AST/U.cr (U/gU.cr) & 5 & 13.2 & 3 & 9.1 & $\mathrm{p}=0.438$ \\
U.ALT/U.cr (U/gU.cr) & 1 & 2.6 & 5 & 15.2 & $\mathrm{p}=0.071$ \\
U.ALP/U.cr (U/gU.cr) & 1 & 2.6 & 9 & 27.3 & $\mathrm{p}=0.003$ \\
U.y-GT/U.cr (U/gU.cr) & 1 & 2.6 & 6 & 18.2 & $\mathrm{p}=0.035$ \\
U.LDH/U.cr (U/gU.cr) & 0 & 0.0 & 12 & 36.4 & $\mathrm{p}<0.001$ \\
U.TP/U.cr (mg/gU.cr) & 1 & 2.6 & 6 & 18.2 & $\mathrm{p}<0.001$ \\
U.Malb/U.cr (mg/gU.cr) & 1 & 2.6 & 1 & 3.0 & $\mathrm{p}=0.717$ \\
U.Si/U.cr (mg/gU.cr) & 1 & 2.6 & 3 & 9.1 & $\mathrm{p}=0.255$ \\
\hline
\end{tabular}


Prevalence of the values above the upper limit of the $95^{\text {th }}$ confidence range. ${ }^{\mathrm{a}} \mathrm{p}$ less than 0.05 for referentmale workers compared to silica exposed male workers.

The effect of work duration on proteinuria and enzymuria on silica exposed male ind ustrial worker: Table 3 shows the effects of work duration on proteinuria and enzymuria on silica exposed male industrial workers. The results shows that: referent male workers with work duration of less than ten years had a significantly shorter work duration and were younger compared to referent male workers with work duration of more than ten years; referent male workers with work duration of less than ten years had a significantly longer work duration, decreased U.TP( $p<0.001)$, U.Malb ( $p=$
0.003), U.ALP ( $\mathrm{p}=0.003), \mathrm{U} . \mathrm{LDH}(\mathrm{p}<0.001)$ compared to silica exposed male industrial workers with work duration of less than ten years; referent male workers with work duration of more than ten years had a significantly shorter work duration, decreased U.TP $(p=0.016)$, U.Malb $(p<0.001)$, U.ALP $(p=0.009)$, U. $\gamma$-GT $(p<0.001)$ and U.LDH $(p<0.001)$ compared to silica exposed male industrial workers with work duration of more than ten years; silica exposed male industrial workers with work duration less than ten years had a significantly shorter work duration and were younger and significantly reduced U.y-GT compared to silica exposed male industrial workers with work duration of more than ten years.

Table 3

Effects of work duration on proteinuria and enzymuria on silica exposed male industrial workers

\begin{tabular}{|c|c|c|c|c|}
\hline \multirow[t]{3}{*}{ Parameter } & \multicolumn{2}{|c|}{ Referent male workers } & \multicolumn{2}{|c|}{ Silica exposed male workers } \\
\hline & $<10$ & $>10$ & $<10$ & $>10$ \\
\hline & years $(n=17)$ & years $(n=21)$ & years $(n=13)$ & years $(n=20)$ \\
\hline \multicolumn{5}{|l|}{ Age (years) } \\
\hline Median & 32.40 & $43.67^{a}$ & 29.00 & $47.33^{\mathrm{d}}$ \\
\hline Range & $27-53$ & $30-53$ & $21-52$ & $32-56$ \\
\hline \multicolumn{5}{|c|}{ Work duration (years) } \\
\hline Median & 7.33 & $20.67^{a}$ & $2.83^{b}$ & $25.57^{\mathrm{dc}}$ \\
\hline Range & $3-10$ & $12-30$ & $1-6$ & $11-31$ \\
\hline \multicolumn{5}{|c|}{ Glomerular integrity } \\
\hline \multicolumn{5}{|c|}{ U. TP/U.cr(mg/gU.cr) } \\
\hline Median & 21.39 & 25.71 & $53.49^{\mathrm{b}}$ & $46.92^{c}$ \\
\hline Range & $5.39-72.16$ & $4.84-186.90$ & $18.61-378.20$ & $15.09-2156.00$ \\
\hline \multicolumn{5}{|c|}{ U.Malb/U.cr(mg/gU.cr) } \\
\hline Median & 1.13 & 0.74 & $10.33^{b}$ & $18.99^{c}$ \\
\hline Range & $0.41-43.35$ & $0.40-111.24$ & $0.62-65.99$ & $1.58-140.02$ \\
\hline \multicolumn{5}{|c|}{$\begin{array}{l}\text { Proximal tubular integrity } \\
\text { U.ALP/U.cr(IU/gU.cr) }\end{array}$} \\
\hline Median & 2.02 & 1.49 & $4.24^{\mathrm{b}}$ & 2.92 \\
\hline Range & $0.00-5.99$ & $0.00-11.43$ & $2.01-9.65$ & $0.65-32.02$ \\
\hline \multicolumn{5}{|c|}{ U. $\gamma$-GT / U.cr(IU / gU.cr) } \\
\hline Median & 36.32 & 32.25 & 49.67 & $69.02^{\mathrm{dc}}$ \\
\hline Range & $21.47-122.14$ & $13.72-103.83$ & 29.43-111.02 & $33.58-490.90$ \\
\hline \multicolumn{5}{|c|}{ U.LDH/U.cr(IU/gU.cr) } \\
\hline Median & 24.98 & 23.79 & $49.25^{b}$ & $57.62^{c}$ \\
\hline Range & $18.83-50.92$ & $16.89-54.03$ & 30.34-99.61 & $27.96-410.86$ \\
\hline \multicolumn{5}{|c|}{ U.AST / U.cr(IU / gU.cr) } \\
\hline Median & 7.80 & 8.91 & 8.05 & 10.18 \\
\hline Range & $4.54-22.96$ & $2.48-30.72$ & $1.26-20.61$ & $4.96-88.04$ \\
\hline \multicolumn{5}{|c|}{ U.AL T/U.cr(IU/gU.cr) } \\
\hline Median & 7.49 & 6.34 & 6.89 & 6.97 \\
\hline Range & $0.00-16.70$ & $0.00-18.29$ & $0.00-17.93$ & $2.64-53.76$ \\
\hline \multicolumn{5}{|c|}{ U.Si/U.cr(mg/gU.cr) } \\
\hline Median & 6.47 & 5.33 & 6.35 & 4.96 \\
\hline Range & $0.00-13.38$ & $0.58-17.43$ & $1.15-36.80$ & $1.20-17.02$ \\
\hline
\end{tabular}


Results are expressed as Median (Range). ${ }^{\mathrm{a}} \mathrm{p}<0.05$ for comparison between referent male workers with work duration of less than 10 years versus referent male workers with work duration of more than 10 years; ${ }^{c} p<0.05$ represents comparison between referent male workers with work duration of less than 10 years and silica exposed male industrial workers with work duration of less than 10 years;
${ }^{c} p<0.05$ represents comparison between referent male workers with work duration of more than 10 years and silica exposed male industrial workers with work duration of more than 10 years; ${ }^{d} p$ less than 0.05 represents silica exposed male industrial workers with work duration of $<10$ years and silica exposed male industrial workers with work duration of more than 10 years.

Table 4

Significant Spearman's correlation coefficient among the measured parameters among silica exposed and referent male workers

\begin{tabular}{|c|c|c|c|}
\hline Parameter & Parameter & Referent $(\mathrm{n}=38)$ & Silica exposed $(n=33)$ \\
\hline \multirow[t]{4}{*}{ Age } & Work duration & $\mathrm{r}=0.782$ & $\mathrm{r}=0.832$ \\
\hline & & $\mathrm{p}<0.001$ & $\mathrm{p}<0.001$ \\
\hline & U.Si & & $\mathrm{r}=-0.376$ \\
\hline & & & $\mathrm{p}=0.031$ \\
\hline \multirow[t]{6}{*}{ U.AST } & U.ALT & & $\mathrm{r}=0.574$ \\
\hline & & & $\mathrm{p}<0.001$ \\
\hline & U. $\gamma$-GT & $\mathrm{r}=0.331$ & $\mathrm{r}=0.547$ \\
\hline & & $\mathrm{p}=0.043$ & $\mathrm{p}=0.001$ \\
\hline & U.LDH & $\mathrm{r}=0.577$ & \\
\hline & & $\mathrm{p}<0.001$ & \\
\hline \multirow[t]{2}{*}{ U.ALT } & U.LDH & $\mathrm{r}=0.364$ & \\
\hline & & $\mathrm{p}=0.025$ & \\
\hline \multirow[t]{4}{*}{ U.ALP } & U.TP & & $\mathrm{r}=0.358$ \\
\hline & & & $\mathrm{p}=0.041$ \\
\hline & U.LDH & $\mathrm{r}=0.625$ & \\
\hline & & $\mathrm{p}<0.00 \mathrm{I}$ & \\
\hline \multirow[t]{2}{*}{ U. $\gamma-G T$} & U.LDH & $\mathrm{r}=0.529$ & $\mathrm{r}=0.484$ \\
\hline & & $\mathrm{p}=0.001$ & $\mathrm{p}=0.004$ \\
\hline \multirow[t]{4}{*}{ U.LDH } & U.TP & & $\mathrm{r}=0.596$ \\
\hline & & & $\mathrm{p}<0.001$ \\
\hline & U.Malb & $\mathrm{r}=0.383$ & $\mathrm{r}=0.491$ \\
\hline & & $\mathrm{p}=0.018$ & $\mathrm{p}=0.004$ \\
\hline \multirow[t]{2}{*}{ U.TP } & U.Malb & $\mathrm{r}=0.628$ & $\mathrm{r}=0.557$ \\
\hline & & $\mathrm{p}<0.001$ & $\mathrm{p}=0.001$ \\
\hline
\end{tabular}

Relationship between work duration and age and the other urinary measured parameters: Table 4 shows significant spearman's correlation coefficient among the measured parameters in the referent male workers and silica exposed male industrial workers. Results show that: among the referent non-smoking male industrial workers, significant correlation was observed between age and each of work duration (for both the referent and silica exposed) and U.Si (negatively) (for silica exposed); U.AST and each of U.ALT (for silica exposed), U. $\gamma-$
GT (for both the referent and silica exposed), U.LDH (for referent); U.ALT and U.LDH (for referent); U.ALP and each of U.TP (for silica exposed), and U.LDH (for referent); U. $\gamma$-GT and U.LDH (for both referent and silica exposed); U.LDH and each of U.TP (for silica exposed), and U.Malb (for both referent and silica exposed); U.TP and U.Malb (for both referent and silica exposed). There was no significant correlation between silica levels and work duration in silica exposed male industrial workers. 


\section{DISCUSSION}

Results of significantly increased U.TP and U.Malb in silica exposed male industrial workers compared to referent male workers suggest that there is an association between silica exposure and glomerular injury. This could be due to epithelial cell damage that results in alteration in the molecular integrity of the sialoprotein. Sialoprotein is a polyanionic protein that covers the glomerular epithelial into urinary space $(14,15)$. These findings are in line with those of other investigators who reported that silica exposure resulted in elevation of U.Malb and transferrin (high molecular weight proteins) among silica exposed industrial workers $(3,16,17)$. The significantelevation of U.ALP, U. $\gamma$-GT and U.LDH in silica exposed male industrial workers compared to referent male workers suggest renal proximal damage and agrees with the findings of other investigators who reported significantly increased U .RBP (biomarker for renal proximal tubular dysfunction) and U.NAG among silica exposed industrial workers $(3,16,17)$. The observed significant correlation between U.TP and each of U.LDH $(\mathrm{p}<0.001)$ and U.ALP $(\mathrm{p}<0.001)$ supports the suggestion that silica exposure results in disruption of both the glomerular integrity and proximal tubular structural integrity.

The observed similar levels of U.Si levels in both the referent and silica exposed maleindustrial workers suggest similar silica exposure levels. However, the glomerular and proximal tubular injury observed in the silica exposed male industrial workers but not in the referent male workers could be due to the type and freshness of silica particles that the two study groups are exposed to. The referent male workers could be exposed to amorphous aged silica particles with low toxicity while the silica exposed male industrial workers could be exposed to fresh crystalline silica generated during the processing of ceramics, bricks and tiles with high toxicity (18).

The observed absence of a correlation between work duration and U.Si levels may imply that injury due to silica exposure is not duration related. It may also imply that it is non-progressive in nature in that it remains subclinical. This observation is in line with other investigators who observed no correlation between work duration and either U.Si or any of the studied urinary parameters of kidney function among silica exposed male industrial workers (14, 16). In contrast, other investigators (3) demonstrated correlation between work duration and U.Si levels, as well as each of the- measured urinary parameters of kidney function (U.TP, U.Malb, U.RBP, U.NAG, and U.GST) among silica exposed male industrial workers while work duration only correlating with U.GST among silica exposed male industrial workers (3). Correlations between parameters for proximal tubular structural integrity and between parameters for glomerular integrity either in the referent or silica exposed industrial workers or both were difficult to explain. The observed negative correlation between age and U.Si levels in silica exposed industrial workers suggests that silicon levels decrease with age. This observation is in line with the higher nonsignificant levels of U.Si in younger silica exposed male industrial workers with work duration of less than ten years compared to older silica exposed male industrial workers with work duration of more than ten years.

In conclusion, the present study shows: that silica exposure is associated with glomerular and proximal tubular injury which is not work duration related and seemingly subclinical and nonprogressive; that urinary silica levels seem similar in all groups and are not affected by exposure and work duration: the reason for this observation is unclear.

\section{ACKNOWLEDGEMENTS}

Wegreatly appreciate the expert advice and guidance ofDr. A. A. Amayo, Department of Clinical Chemistry and Dr. B. Ndungu, Department of Human Anatomy, University of Nairobi in the early stages of this study. Special thanks go to the management, colleagues and all the staff of Kenyatta National Hospital and especially to staff members who volunteered their urine samples for use as the referents. Thanks to Mr.S. K. Waithaka of Clinical Chemistry together with Mrs. P. Onyono and Mr. N. Njeru both of Hichem Company Limited for assisting in the running the samples with the Olympus AU 640® fully automated Clinical Chemistry Analyzer in the Clinical Chemistry section. The technical support given by Mr D. Andala, Mr. D. Osoro, M. Onkoko of the Chemistry Department, Kenyatta University and Mr. P. Chepkuon of the Department of Mines and Geology, Ministry of Natural Resources in the analysis of urinary silicon using the Buck Model 210 VGp® Atomic Absorption Spectrophotometer is greatly appreciated. We also thank Mr. M. King' ori of Clayworks Bricks and Tiles Factory and all their factory staff for giving their urine samples for the silica exposed male study subjects. We are most grateful to Mr. E. Muniu and G. M. Ngondi both of Kenya Medical Research Institute, Centre for PublicHealth Research and KEMRI GraduateSchool, respectively for Statistical Analysis. We appreciate the Computer assistance and support given by $\mathrm{Mr}$. S. Mwangi of Railway Training Institute.

\section{REFERENCES}

1. Graham, W.G.B. Silicosis. Clin. Chest. Med. 1992; 13: 253-267.

2. Collis, E. I. Astudy of mortality of coal miners. England and Wales. J. Ind. Hyg. 1922; 4: 307-331. 
3. EL-Safty, I.A.M., GadalIah, M., Shouman, A.E. and Nessim, D.E. Subclinical nephrotoxicity caused by smoking and occupational silica exposure among Egyptian industrial workers. Arch. Med. Res. 2003; 34: 415-421.

4. Ivandic, M., Hofmann, W. and Guder, W.G. The use of knowledge-based systems to improve medical knowledge about urine analysis. Clin. Chim. Acta. 2000; 297: 251-260.

5. Lauwerys, R., Bernard, A. and Cardenas, A. Monitoring of early nephrotoxicity effects of industrial chemicals. Toxicol. Lett. 1992; 64: 65N33-N42.

6. Jung, K. and Burchardt, U. Urinary enzymes in research and in clinical medicine. J. Clin. Chem. Clin. Biochem. 1987; 25: 823-828.

7. Stetinova, V., Trejtnar, F., Kopecky, J. et al. Possible use of excretion of tubular epithelial cells for the study of the nephrotoxic effect of xenobiotics. J. Pharm. Tox. Met. 1996; 36: 199-204.

8. Melo, D.A.S., Saciura, V.C., Oliveira, C.S.A. et al. Evaluation of renal enzymuria and cellular excretion as a marker of acute nephrotoxicity due to an overdose of paracetamol in Wistar rats. Clin. Chim. Acta. 2006; 373: 88-91.

9. Mann, C.J. Observational research methods. Research design II: cohort, cross sectional and case-control studies. Emerg. Med. J. 2003; 20: 54-60.

10. Lison, D., Lardot, C., Huaux, F., Zanetti, G. and Fubini, B. Infuence of particle surface area on the toxicity of insoluble manganese dioxide dusts. Arch. Toxicol. 1999; 71: 725-729.
11. Woolerton, J., Jary, D.R., Dunn, P.J. and Speed, J.F. Urine albumin/creatinine ratio and clinical correlates in a diabetic population. N. Z. Med. J. 1987; 100: 130134.

12. Jung, K. Enzyme activities in urine. How should we express their excretion? Eur. J. Clin. Chem. Clin. Biochem. 1991; 29: 725-729.

13. Barratt, J., and Topham, P. Urine proteomics: the present and future of measuring urinary protein components in disease. Can. Med. Assoc. J. 2007; 177: 361-368.

14. De-Bats, A., Gordon, A.H. and Rhodes, E.L. Variation in glomerular sialic acid content in diabetes and the result of aging. Clin. Sci. Mol. Med. 1974; 47: 93-95.

15. Chang, R.L., Deen, W.M., Robertson, C.R. and Brenner, B.M. Perm selectivity of the glomerular capillary wall: III. Restricted transport of polyanions. Kidney Int. 1975; 8: 212-218.

16. Boujemaa, W., Laywerys, R. and Bernard, A. Early indicators of renal dysfunction in silicotic workers. Scand. J. Work Environ. Health. 1994; 20: 180-183.

17. Hotz,P., Gonzalez-Lorenzo, J., Siles, E. et al.Subclinical signs of kidney dysfunction following short exposure to silica in the absence of silicosis. Nephron. 1995; 70: 438-442.

18. Stratta, P., Canavese, C., Messuerotti, A., Fenoglio, I. and Fubini, B. Silica and renal diseases: no longer a problem in the 21st century? J. Nephrol. 2001; 14: 228247. 